\title{
Diversidad, conflictos, confianza y aprendizaje social en la Gobernanza Colaborativa. Un estudio de caso en las Comisiones Regionales de Uso de Borde Costero
}

Diversity, conflicts, trust and social learning in Collaborative Governance. A case study in the Regional Coastal Use Commissions

María José Benavente Bargetto *

Resumen: Las Comisiones Regionales de Uso de Borde Costero (CRUBC) son un arreglo de gobernanza colaborativa a nivel local subnacional para gestionar el borde costero chileno. Están compuestas por actores públicos de diferentes niveles y actores no estatales, con presencia en cada una de las regiones con acceso al mar chileno.

El objetivo central de este estudio es analizar la interacción social de los miembros de la Comisión Regional de uso de Borde Costero de la Región del Bío-Bío y cómo este contribuye o dificulta los arreglos de gobernanza colaborativa en el marco de los conflictos socio territoriales del borde costero.

Dada la relevancia del borde costero como territorio natural y estratégico de nuestro país, es menester indagar en los procesos de gobernanza colaborativa que se han propuesto como modelos de gestión necesarios y útiles para resolver problemas públicos en territorios de conflictividad latente y donde están en juego recursos naturales de alto valor para la comunidad.

Para el análisis se propone un enfoque innovador de gobernanza colaborativa que se centra en la interacción de los miembros, superando las predisposiciones positivas de la diversidad de actores y la inclusión de miembros no estatales en la resolución de problemas públicos. Los resultados arrojan que en las Comisiones Regionales de Uso de Borde Costero no existe un aprendizaje social considerable entre los miembros ya que se presentan obstáculos que impiden la concreción de misiones conjuntas y una visión integrada del borde costero.

Palabras clave: gobernanza colaborativa, borde costero, interacción social, aprendizaje social, diversidad.

\begin{abstract}
The Regional Coastal Use Commissions (CRUBC) are a collaborative governance arrangement at the local sub-national level to manage the Chilean coastline. They are made up of public actors of different levels and non-state actors, with a presence in each of the regions with access to the Chilean sea.

The main objective of this study is to analyze the social interaction of the members of the Regional Commission for the use of the Coastal Edge of the Bío-Bío Region and how this contributes or hinders collaborative governance arrangements in the framework of the socio-territorial conflicts of the coastal edge.
\end{abstract}

\footnotetext{
* Magíster en Política y Gobierno, Universidad de Concepción. Investigadora asociada programa Convergencia Regionalista de Estudios Aplicados (CREASUR), Universidad de Concepción, marbenavente@udec.cl
} 


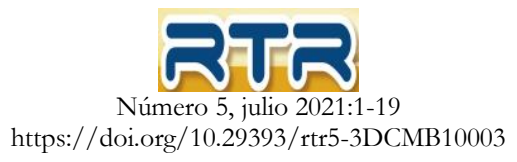

Given the relevance of the coastline as a natural and strategic territory of our country, it is necessary to investigate the collaborative governance processes that have been proposed as necessary and useful management models to solve public problems in territories of latent conflict and where resources are at stake. natural resources of high value to the community. For the analysis, an innovative collaborative governance approach is proposed that focuses on the interaction of members, overcoming the positive predispositions of the diversity of actors and the inclusion of non-state members in the resolution of public problems.

The results show that in the Regional Commissions for the Use of the Coastal Edge there is no considerable social learning among the members since there are obstacles that prevent the realization of joint missions and an integrated vision of the coastline.

Keywords: collaborative governance, coastline, social interaction, social learning, diversity.

Recibido: 3 octubre 2020 Aceptado: 19 abril 2021

\section{Introducción}

La gobernanza se ha transformado en un concepto amigable que ha puesto nuevas esperanzas en los desafíos de la modernidad. La sociedad actual se ha vuelto cada vez más compleja por una serie de razones, entre ellas, la democratización, que ha puesto nuevas ambiciones y expectativas en la ciudadanía.

Actualmente las personas son y quieren ser ciudadanos cada vez más activos en cuanto a las decisiones y soluciones que se adopten en la resolución de los problemas que les incumben. Por otro lado, el mundo vive un importante cambio de paradigma que ha puesto a la naturaleza como elemento prioritario a considerar en el debate público, lo que, a su vez, ha insertado en el juego político a otros actores que antes quedaban relegados.

La gestión de los recursos naturales y del territorio es relevante, ya no solo desde una perspectiva economicista, sino también social, cultural y medioambiental. En este sentido, el borde costero es un espacio territorial estratégico y geográficamente relevante para Chile.

Si existe un espacio de disputa territorial para diversos fines, es el borde costero, ya que, en él, se realizan diversas actividades económicas, como la minería, el turismo, la acuicultura, la pesca, entre otras y también, es un espacio delicado y sensible a las alteraciones del ecosistema. Existen, además asentamientos humanos que habitan en este espacio, muchos de los cuales se reconocen como ancestrales.

La gestión del borde costero es compleja y puede llegar a verse implicada inclusive a cosmovisiones distintas del mundo. En este contexto, la resolución de los problemas en un afán moralmente democrático, no puede más que, involucrar a la multiplicidad de actores para poder obtener compromisos conjuntos y soluciones adecuadas que se adapten a los distintos intereses en disputa.

La gobernanza colaborativa se presenta como un mecanismo que permite resolver estas tareas complejas, es decir, cuando las soluciones son inciertas o controvertidas, cuando las dimensiones que componen un problema involucran a diversos sectores y perspectivas disciplinarias o cuando existen distintas creencias sobre cómo se debe manejar un problema. 


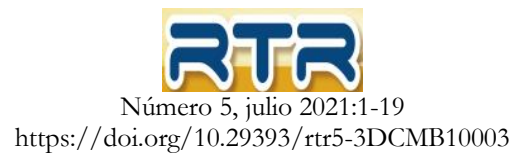

Se ha erigido un discurso desde el enfoque de la gobernanza que promueve la participación y el involucramiento de los distintos sectores de la sociedad para así poder enfrentar los problemas públicos con una perspectiva sistémica, obteniendo resultados mucho más sustentables en el tiempo.

La justificación de esta definición de gobernanza y su promoción, está alineado a los valores democráticos, pero con una sobrecarga al elemento de la participación. El autor Kooiman citado en Canto (2012) señala que la amplia participación societal en la gobernanza es una expresión de la democracia y por ende es un estado deseable de los asuntos:

Como un juicio de hecho, el enfoque de la gobernanza interactiva sostiene que muchos actores en diferentes posiciones y niveles de la sociedad están involucrados en la gobernanza. Pero también hay un lado normativo en la ecuación, la comprensión de que la amplia participación societal en la gobernanza es una expresión de la democracia y, por lo tanto, un estado deseable de los asuntos (p.337).

Lo controversial, es que, por recargar un énfasis en la participación y la diversidad de actores, se obvian cuestiones como, el desequilibrio de poderes, la eficacia de la rendición de cuentas, la representatividad real de actores no estatales, la superposición de intereses privados por sobre el interés general, entre otros, pudiendo incluso agravar los conflictos o promover el nacimiento de otros que no existían.

En atención a lo anterior, es preciso ahondar en los procesos colaborativos para distinguir los elementos que pueden contribuir u obstaculizar los arreglos de gobernanza y observar los matices, teniendo cuidado con considerar una predisposición siempre positiva del check list de una gobernanza deseable.

Siddiki, Kim, \& Leach (2017) realizaron un estudio que presta atención detenidamente a los efectos de la diversidad en los resultados de la gobernanza. Establecen que no todo arreglo de gobernanza produce los resultados óptimos, y demuestran que la diversidad de creencias y la diversidad de afiliación de los participantes son dos variables que inciden en el aprendizaje social. Este último es un resultado relevante en la interacción colaborativa, ya que ésta es la base para la acción conjunta.

De esta manera, la diversidad puede implicar desafíos y oportunidades para arreglos efectivos en procesos colaborativos de gobernanza, ya que ésta es a su vez, un elemento clave para el aprendizaje social.

Las CRUBC desde 1997, forman parte de una red institucional que se ocupa de administrar el borde costero nacional, donde intervienen una diversidad de confluencias. Estas proponen impulsar, autorizar, administrar y fiscalizar las actividades humanas que involucran los terrenos de playas fiscales, la playa, las bahías, golfos, estrechos y canales interiores, y el mar territorial de la República.

Alojadas en los Gobiernos Regionales del país, exceptuando la Región Metropolitana, las CRUBC, tienen la responsabilidad de implementar la Política Nacional de Uso de Borde Costero y otras funciones como aprobar o rechazar las concesiones marítimas y aprobar, rechazar o proponer modificaciones fundadas al espacio costero marino solicitado por comunidades. Además, las CRUBC deben propender a elaborar una Zonificación de Borde Costero.

Se presentan las CRUBC, como un buen estudio de caso para el análisis de la gobernanza, puesto que, se componen por actores diversos con distintos puntos de vista e intereses sobre la franja costera y, además, se ubican en diferentes niveles de gobierno y sectores de la sociedad civil y el mercado. Por otra parte, las Comisiones están llamadas a resolver problemas complejos, que pueden ser políticos, técnicos e interdisciplinarios.

El objetivo de este estudio es analizar el funcionamiento de la CRUBC (2019-2020) y los efectos producidos en el aprendizaje social de sus miembros como resultado de los procesos del modelo de gobernanza colaborativa. considerando para el análisis, los hallazgos de la investigación de 
Siddiki, et al., (2017) y el modelo propuesto de Ansell y Gash (2007). A partir de los resultados empíricos en el estudio de la gobernanza, el propósito de la presente investigación es contribuir a la gestión costera y al futuro de las CRUBC, mediante recomendaciones dirigidas a mejorar la calidad de la gobernanza colaborativa.

\section{Gobernanza Colaborativa: significado y dimensiones}

El concepto de gobernanza nace como un cambio paradigmático en la forma de gobernar después de los años 90 y que supone una mayor horizontalidad de las decisiones gubernamentales. Se incorpora y visibiliza a otros actores antes excluidos u ocultos y da cuenta del cambio del rol del Estado con respecto a la relación con la sociedad y el mercado, planteando que los gobiernos se hacen cada vez más dependientes de la cooperación y de los recursos de actores privados y sociales, fuera de su control jerárquico, para buscar soluciones a los problemas públicos.

Lo que se requiere es otro proceso de gobernar, un proceso de gobierno en modo postgubernamental que incluya tanto la acción del gobierno como la de los ciudadanos, que conjunte e integre las capacidades y recursos del gobierno con los de las empresas económicas, las organizaciones de la sociedad civil y los centros de información y conocimiento. La nueva estructura del proceso de gobernar, relacional, asociada, produce legitimidad política, aceptación social y eficacia directiva y hace que el gobierno reconstruya con un nuevo proceso y un nuevo rol su capacidad directiva (Aguilar, 2014, p.17)

En ese sentido, la perspectiva de gobernanza nos permite observar y analizar nuevas transformaciones en el Estado, y en las políticas públicas, como también nos ofrece una pauta de acción para organizar la administración pública en base a nuevos modelos de gestión que permitirían elaborar instrumentos que promuevan un escenario más proclive a la participación de diversos actores sociales, relaciones multinivel, a la horizontalidad de los procesos, la rendición de cuentas y la transparencia.

Brugué, Canal y Paya (2012), evidencian este cambio paradigmático, donde el modus operandis administrativo clásico que pone énfasis en la racionalidad tecnocrática, se encuentra desbordado al verse enfrentado a una realidad compleja y multidimensional, causando una presión en las administraciones y un descontento social.

Pero más allá de las causas ${ }^{1}$, lo cierto es que, existen nuevas propuestas y enfoques que intentan analizar estas nuevas modalidades. Por ejemplo, Kooiman et al. (2005) desarrolla el concepto de gobernanza interactiva, dotándole de especial importancia a las interacciones sociales, ya que éstas serían la forma de enfrentar la diversidad, complejidad y dinamismo de las sociedades modernas.

Surge también, el concepto de colaboración que se ha ido incorporando a la gestión pública en la medida que éstas se vuelven más complejas e interdependientes.

De esta manera, más positiva, se podría argumentar que las tendencias hacia la colaboración también surgen del crecimiento del conocimiento y la capacidad institucional. A medida que los conocimientos se especializan y distribuyen cada vez más y que las infraestructuras institucionales se vuelven más complejas e interdependientes, aumenta la demanda de colaboración (Ansell \& Gash, 2007, p.2).

\footnotetext{
${ }^{1}$ Las causas de la aparición de la gobernanza dan lugar al debate de las últimas décadas sobre las transformaciones del estado, en los cuales han confluido factores muy diversos. Para ahondar en este tema se puede leer a Natera (2005) http://www.scielo.org.mx/scielo.php? script=sci arttext\&pid=S0188-25032005000400004
} 
La colaboración implica alcanzar objetivos comunes, a menudo trabajando a través de fronteras y en relaciones multisectoriales y de múltiples actores. La colaboración se basa en el valor de la reciprocidad y puede incluir al público (Leary \& Vij, 2012).

Ansell y Gash (2007) apuntan a una definición concreta de gobernanza colaborativa. Para ellos la gobernanza colaborativa es:

Un acuerdo de gobierno en el que uno o más organismos públicos involucran directamente a las partes interesadas no estatales en un proceso colectivo de toma de decisiones, que es formal, orientado al consenso, deliberativo y que tiene por objeto elaborar o aplicar políticas públicas o administrar programas o bienes públicos (p.2)

Esta definición es más restrictiva que otras que aparecen en la literatura y considera seis condiciones clave: (1) el foro es iniciado por organismos e instituciones públicas, (2) los participantes en el foro incluyen actores no estatales, (3), los participantes participan directamente en la toma de decisiones y no son "meramente" consultados por los organismos públicos, (4) el foro se organiza formalmente y se reúne colectivamente, (5) el foro tiene por objeto tomar decisiones de consenso, (6) el enfoque de la colaboración se centra en la política pública o la gestión pública y no en hechos puntuales de negociación o mediación, así como también, es necesario que, las partes tomen decisiones durante el proceso y no solo recoger meras consultas para terminar confiando en los expertos.

Sin embargo, la colaboración puede contribuir a malas políticas o a buenas políticas. Tal como señala Wanna (2008):

La colaboración suele atraer un "giro" positivo. A menudo se ve como algo positivo: la colaboración es mejor, es creativa, transformativa e implica resultados beneficiosos. Sin embargo, hay otras dimensiones que deben considerarse. Los esfuerzos de colaboración pueden implicar el logro de algún resultado o, alternativamente, la negación o prevención de que algo suceda. Podemos colaborar para fines "buenos" y para fines "malos" (p.3).

En este sentido, cuando la relación se vuelve colaborativa, resulta pertinente el análisis empírico de las generalizaciones y las teorías que se han ido construyendo. Así, por ejemplo, el estudio de Siddiki, Kim \& Leach (2017) propone como variables relevantes la diversidad, la confianza y el aprendizaje social, concluyendo que aquellas variables si están relacionadas con los resultados de gobernanza.

Por otra parte, Ansell y Gash (2007) han señalado que las condiciones presentes al comienzo de la colaboración pueden desalentar la cooperación entre las partes interesadas toda vez que haya asimetría de poder, recursos y/o conocimientos. Así como también, carencia de incentivos y limitaciones a la participación y, por último, los antagonismos y conflictividad del pasado también serían una condicionante para la colaboración. Otros factores relevantes, son el diseño institucional y el liderazgo.

Estos elementos van complejizando la sencilla ecuación de más participación es mejor, un imperativo democrático que tiende a nublar la realidad y a relajar los esfuerzos de gestión que deberían intentar colocar los "ingredientes" en su justa medida para propender a soluciones de problemas públicos de manera integral y sostenibles con las comunidades y la naturaleza.

\section{El Aprendizaje Social como resultado de la Gobernanza Colaborativa}

La teoría revisada en el estudio de Siddiki, Kim \& Leach (2017) arroja que uno de los resultados sobresalientes en los contextos de colaboración son el aprendizaje social, ya que las personas 
que participan en procesos deliberativos con otros conocedores de diferentes orígenes y se les presenta información relacionada con el trabajo que se está llevando a cabo, tienen la probabilidad de estar expuestos a nuevas ideas e información y de potenciar y forjar nuevas relaciones sociales.

El aprendizaje social es definido por Schusler, Decker y Pfeffer (citado en Siddiki, Kim, \& Leach, 2017), como "el aprendizaje que ocurre cuando las personas se involucran entre sí, compartiendo diversas perspectivas y experiencias para desarrollar un marco común para la comprensión y la base para la acción conjunta" (p.864).

Es posible distinguir dos tipos de aprendizaje: el cognitivo y relacional. El primero son las transferencias de conocimiento y, el segundo son los resultados de las relaciones interpersonales o interacción social (Siddiki, Kim, \& Leach, 2017).

\section{La diversidad y la confianza interpersonal}

Un problema identificado en el análisis de la gobernanza, como ya se ha mencionado, es comenzar el análisis partiendo de suposiciones positivas de los contextos participativos sesgando o encubriendo los verdaderos efectos de la participación.

Lo anterior no es un intento para negar la participación, ya que como señala Kooiman (citado en Ramírez, 2011) "Ningún actor por sí solo, público o privado tiene el conocimiento y la información necesarios para solventar problemas complejos dinámicos y se liga a la necesidad de actuar de manera diversificados" (p.132).

En este sentido, "la gobernanza debería alentar la participación de actores públicos" (Ramírez, 2011, p. 132). Sin embargo, es necesario también señalar que la diversidad de actores, consecuencia posible de la participación, puede tener efectos tanto positivos como negativos en el aprendizaje social de los participantes.

En un contexto de interacción social, la diversidad según la revisión teórica del estudio de Siddiki, Kim, \& Leach (2017) puede tener los siguientes aspectos positivos en entornos complejos de política y gestión:

- Aporta recursos y experiencias que contribuyen a informar sobre tareas y cuestiones técnicas.

- Puede conducir a enfoques holísticos y contextualmente apropiados para reconocer cómo las políticas y los programas afectan a las diferentes partes interesadas.

- Facilita la comprensión compartida de problemas y soluciones de políticas públicas.

Pero la diversidad también puede significar grandes desafíos y ser un obstáculo para el aprendizaje social.

En el ámbito organizacional, la gestión de la diversidad ha sido un tema relevante. Ésta, se ha propuesto como una iniciativa para aumentar las ventajas competitivas, en la medida que las políticas de recursos humanos estén orientadas no solo a promover la diversidad, sino que también a gestionarla (Morales, 2009). En consecuencia, a pesar de la legitimidad que la diversidad otorga al propio proceso, por el hecho de incluir actores disímiles no se garantizan buenos resultados.

Esta inconsistencia teórica sobre la relación entre la diversidad y el aprendizaje, motivan una mayor investigación sobre cómo los diferentes tipos de diversidad influyen en el aprendizaje y también plantean preguntas sobre qué factores podrían moderar la relación entre estos elementos (Siddiki, Kim \& Leach, 2017).

Es aquí donde toma relevancia la hipótesis de Tsai (citado Siddiki, Kim, \& Leach, 2017) quien señala que la confianza amortigua los efectos negativos de la diversidad en el intercambio de 
conocimientos, al reducir la inseguridad y favorecer a una identidad unificada entre los miembros colaboradores (p.865).

En este sentido, la confianza es prometedora en cuanto a facilitar la adquisición de conocimientos y el establecimiento efectivo de redes, es decir, puede beneficiar el aprendizaje cognitivo y relacional. Para analizar la influencia de la diversidad de participantes esta será modelada como la diversidad de afiliación y la diversidad de creencias.

La diversidad de afiliación se refiere a la membresía de los participantes a alguna organización social o política y la diversidad de creencias corresponde a la forma en cómo los participantes piensan y que paradigmas o visiones de mundo tienen para enfrentar un problema en particular.

\section{Otras variables relevantes: La ecuación no es tan sencilla}

La diversidad no es la única que puede influir en el aprendizaje final en un contexto colaborativo y la confianza tampoco es el único elemento que supuestamente modera esta relación. Asimismo, desde los estudios de la gestión de la diversidad en el ámbito empresarial, señalan que los impactos de ésta dependen de otras variables como, por ejemplo, la naturaleza de la tarea, el estilo del liderazgo, entre otros (Morales, 2009). Según la revisión teórica en el estudio de Siddiki, Kim \& Leach (2017) existen otros factores claves que pueden afectar el aprendizaje:

- Conocimiento científico disponible

- Procedimientos justos

- Liderazgo respetado

- Participación extendida

- Creencias

Estos factores también pueden incidir en los resultados de gobernanza colaborativa. Así, por ejemplo, los estudios han demostrado que los mecanismos de toma de decisión justos podrían influir en la disposición del grupo para trabajar en conjunto. Y un liderazgo respetado por todos los actores, facilitaría los resultados positivos.

Además, establecen que las creencias muchas veces actúan como filtros cognitivos que pueden acrecentar u obstaculizar el aprendizaje. Por último, la participación extendida también aparece como un factor relevante a la hora de establecer confianza, generar más oportunidades para compartir e intercambiar información. Por otra parte, Ansell y Gash (2008), sostienen que la percepción de conflicto es un elemento valioso a considerar en los procesos de gobernanza colaborativa y que éste puede considerarse como obstáculo u oportunidad.

\section{Marco Metodológico}

El estudio es de carácter descriptivo-interpretativo, pues se busca analizar el funcionamiento de la CRUBC (2019-2020) y los efectos producidos en el aprendizaje social en sus miembros como resultado de los procesos del modelo de gobernanza colaborativa. Se identifica la diversidad de los participantes, el análisis de las interacciones sociales que ocurren entre ellos y la determinación de los obstáculos y fortalezas presentes en este proceso.

La metodología de este estudio es mixta, ya que se utilizan métodos cuantitativos y cualitativos de la investigación, potenciando así los resultados de cada una y otorgando a su vez una mayor flexibilidad para aproximarse al objeto de estudio. 


\section{Unidad de muestreo}

Para las unidades de análisis de este estudio se consideraron tres muestras.

a) Actores claves de las CRUBC

En una primera instancia como actores claves a personas vinculadas a la coordinación de las CRUBC en regiones del norte centro y sur del país. Fueron los actores claves de las Regiones de Antofagasta, de Tarapacá, del Bío-Bío y de Aysén que estuvieron dispuestas a ser entrevistadas.

En este caso se ha elegido la muestra con el objetivo de obtener un conocimiento previo de las dimensiones que hacen críticos los procedimientos de gobernanza colaborativa en las CRUBC mediante un grupo específico de casos en distintas regiones del país, con la intención de abordar su dimensión geográfica. La siguiente tabla muestra la cantidad y caracterización de entrevistados por región.

Tabla 1. Ficha caracterización muestras de Actores Claves en la CRUBC

\begin{tabular}{|l|l|l|l|}
\hline $\begin{array}{l}\text { Regiones } \\
\text { consultadas }\end{array}$ & $\begin{array}{l}\text { Sexo del } \\
\text { entrevistado/a }\end{array}$ & $\begin{array}{l}\text { Formación } \\
\text { Profesional }\end{array}$ & $\begin{array}{l}\text { Años de participación en } \\
\text { la CRUBC }\end{array}$ \\
\hline Antofagasta & Masculino & Arquitecto & $\begin{array}{l}\text { Más de 2 años y menos } \\
\text { de 5 }\end{array}$ \\
\hline Tarapacá & Masculino & Geógrafo & Más de 5 años \\
\hline Bío-Bío & Masculino & Geógrafo & Más de 5 años \\
\hline Aysén & Femenino & Biólogo Marino & Más de 5 años \\
\hline
\end{tabular}

b) Miembros de la CRUBC, Región de Bío-Bío

Este estudio consideró a miembros de la CRUBC de la Región del Bío-Bío tanto para la autoaplicación de un cuestionario como la aplicación de entrevistas. Este muestreo tuvo una intencionalidad de conveniencia, por lo que su origen radica principalmente en la accesibilidad para poder acceder a las unidades de análisis.

El cuestionario de auto-aplicación fue enviado a los 49 miembros de la CRUBC registrados en junio 2020. La lista de miembros fue proporcionada por la Oficina Técnica de la CRUBC del Bío-Bío. Un total de 10 respondieron al cuestionario.

Las entrevistas semi-estructuradas fueron aplicadas al sector académico y a gremios del sector empresarial los cuales participan como miembros de la Comisión Regional de Uso de Borde Costero de la Región del Biobío durante el año 2020. Se realizó un total de 3 entrevistas a miembros durante el mes de noviembre del 2020. La siguiente tabla presenta un resumen de la cantidad de entrevistados/as por cada tipo de actor. 
Tabla 2. Ficha caracterización muestra de los miembros de la CRUBC Biobío

\begin{tabular}{|l|l|l|l|}
\hline Tipo de Actor del & $\begin{array}{l}\text { Sexo } \\
\text { entrevistado/a }\end{array}$ & $\begin{array}{l}\text { Años de participación en } \\
\text { CRUBC Bío-Bío }\end{array}$ \\
\hline $\begin{array}{l}\text { Representante sector } \\
\text { pesca en la CRUBC } \\
\text { Bío-Bío }\end{array}$ & Femenino & $\begin{array}{l}\text { Ingeniera Civil } \\
\text { Industrial }\end{array}$ & Más de 5 años \\
\hline $\begin{array}{l}\text { Representante sector } \\
\text { académico en la } \\
\text { CRUBC Bío-Bío }\end{array}$ & Masculino & $\begin{array}{l}\text { Ingeniero } \\
\text { Industrial }\end{array}$ & Civil \\
\hline $\begin{array}{l}\text { Representante sector } \\
\text { portuario en la de 5 años } \\
\text { CRUBC Bío-Bío }\end{array}$ & Masculino & Ingeniero Comercial & $\begin{array}{l}\text { Más de 2 años y menos de 5 } \\
\text { años }\end{array}$ \\
\hline
\end{tabular}

\section{Técnica de recogida de datos}

a) Entrevistas semi-estructuradas

Ahora bien, la técnica utilizada es la entrevista semiestructurada con la finalidad de aportar información relevante sobre el proceso de gobernanza. En las entrevistas a los actores claves, los temas de la pauta fueron: función y rol que cumple en la CRUBC, cantidad de años de participación, procedimiento para incorporación de temas a la agenda/tabla, procedimiento de incorporación de miembros, principales logros de la CRUBC, principales temas de discusión, frecuencia de las reuniones, obstáculos del funcionamiento de la CRUBC, liderazgos visualizados, incidencia de la CRUBC a nivel regional.

En las entrevistas semiestructurada con los miembros del CRUBC Bío-Bío, los temas de la pauta fueron: función y rol que cumple en la CRUBC, cantidad de años de participación, relevancia de su participación en la CRUBC, creencias y conocimientos sobre el borde costero, aprendizajes obtenidos en la CRUBC, conocimientos sobre gobernanza, nudos críticos para el consenso, fortalezas de la CRUBC, liderazgos identificados y deseados y posibilidades de mejora del funcionamiento de la CRUBC.

b) Cuestionario auto-aplicado

Como técnica de recogida de datos cuantitativo, se elaboró un cuestionario virtual de autoaplicación con preguntas cerradas. Consistió de seis secciones: La primera parte solicita información sobre su representación en la Comisión. En la segunda parte se solicita que indique en base a su percepción, el aprendizaje obtenido a lo largo del proceso. En la tercera parte, se le solicita su opinión sobre los demás miembros de la Comisión. En la cuarta parte, se solicita que manifieste su opinión sobre diversas dimensiones del procedimiento llevado a cabo por la Comisión. En la quinta parte, es necesario que indique su postura con respecto a la naturaleza. Finalmente, en la sexta parte, es necesario 
que indique su postura con respecto al conocimiento científico existente en la Comisión. Está técnica proporcionará información relevante para poder confirmar el contenido obtenido de las entrevistas aplicadas, triangulando las respuestas cerradas con los relatos de los actores.

\section{Técnicas de análisis de datos}

a) Entrevistas semi-estructuradas

El análisis de contenido de las entrevistas permite determinar la importancia que los actores le dan a los distintos elementos estructurales de gobernanza colaborativa, así como también comprender cuales son las dinámicas de la interacción social presentes en la CRUBC.

b) Cuestionario auto-aplicado

La estadística descriptiva permite describir la variabilidad existente en los datos levantados. El presente cuestionario incluyó tanto variables nominales como ordinales. Para las principales variables nominales y ordinales, se consideraron tablas de frecuencia y medidas de tendencia central. También se calculó la diversidad de afiliación, la cual se midió utilizando el índice de heterogeneidad de Blau para reflejar cuantos tipos de diferentes actores están representados en cada sector. Las categorías utilizadas para la construcción del índice son las siguientes: Gobierno/Administración Pública, Universidad/Consultor Científico, representantes pesca artesanal/industrial, representantes sector turismo, representantes sector acuícola, pueblos originarios, grupos ambientalistas, otros. La fórmula de Blau se representa con el siguiente índice de heterogeneidad:

$$
1-\Sigma p_{i}^{2}
$$

Donde $p$ es la proporción de miembros en una categoría determinada e $i$ es el número de categorías posibles. A mayor puntuación indica una mayor heterogeneidad y con ello, una mayor diversidad de categorías de actores representados en un sector.

\section{Resultados}

\section{Ámbito y Atribuciones de las Comisiones Regionales de Uso de Borde Costero}

Un primer paso en el análisis fue describir los procedimientos y el rol que tienen las (CRUBC) con respecto a la gestión del borde costero en el país para contrastar con lo planteado en la Política Nacional.

Se presentan coincidencias compartidas en la crítica que hacen los entrevistados con respecto al rol de las CRUBC en la implementación de la Política Nacional de Uso de Borde Costero, señalando que, actualmente el trabajo que realiza la CRUBC es limitado y que no alcanza para ejercer un rol planificador del borde costero.

Según lo informado por los entrevistados/as las CRUBC actualmente centran sus esfuerzos en la aprobación o rechazo de las Concesiones Marítimas del borde costero y sesionan principalmente en base a la demanda de estas solicitudes.

Si bien existe un conocimiento sobre el alcance la Política Nacional de Uso de Borde Costero de 1994 y las funciones de la CRUBC que emanan de esta, señalan que, los esfuerzos van dirigidos 
netamente a resolver las solicitudes de las concesiones marítimas, como una exigencia desde el nivel central de tipo consultivo y no vinculante.

Una de las atribuciones más esperadas de la CRUBC, es la elaboración de una propuesta zonificación del Borde Costero Regional, la cual debe ser presentada a la Comisión Nacional de Uso del Borde Costero para su aprobación. Además de las eventuales modificaciones a la zonificación vigente o en proceso de aprobación.

Solo dos regiones cuentan con una Zonificación de Borde Cotero (Región de Aysén y Coquimbo). Es relevante precisar, que, en particular, la Región del Bío-Bío cuenta con una Zonificación aprobada, pero sin decreto. En este sentido, los actores coinciden en que los instrumentos de planificación territorial, particularmente la Zonificación de Uso de Borde Costero, son fundamentales para poder tener una mayor incidencia en la gestión del borde costero y para tener mayor capacidad de resolución en los problemas que se susciten por diferencias en los usos del borde costero. Pero algunos ven con dificultad poder contar con este instrumento por falta de orientación y recursos para poder aprobarlo, sobre todo ante las nuevas exigencias ambientales que actualmente se requieren para validar los instrumentos de planificación territorial.

Con respecto a la toma de decisiones en las CRUBC, todas estas requieren de aprobación mediante el voto de los miembros, sin embargo, los actores son capaces de reconocer que hay ciertas alineaciones en las decisiones que se concretan, donde se terminan aprobando los proyectos por inercia o influencias de poder más que por un resultado de deliberación al interior de la Comisión. La influencia del intendente en los votos y la aprobación por el Sistema de Evaluación Ambiental Estratégica de los proyectos de inversión, influyen en el comportamiento de los votos de los integrantes de la CRUBC. Con respecto a la incorporación de miembros en la CRUBC, la Política Nacional de Uso de Borde Costero define la lista de miembros, sin embargo, está con el tiempo se ha ido modificando de acuerdo con nuevas problemáticas.

La modificación de la lista de los miembros ha sido por la identificación de nuevas temáticas por la CRUBC, que, previa aprobación del Intendente, realiza una invitación al actor respectivo. También, existen casos donde actores públicos o privados han solicitado ser parte de la CRUBC. En este punto destaca que la Región del Bio-Bío fue la única región de las consultadas donde se ha dado la instancia en que personas han demandado su participación o se han interesado en ser miembros oficiales de la CRUBC, ya que en otras regiones nunca un actor externo ha solicitado interés en ser miembro de la CRUBC.

De acuerdo a la información recolectada, para las cuatro regiones, es posible observar que la agenda temática se va construyendo en base a la solicitud de concesiones marítimas y en menor medida a otras problemáticas. Según las consultas realizadas, no hay impedimento para que los miembros propongan temas para agregarlos en la tabla e incluso existe un mecanismo para realizar sesiones extraordinarias si los miembros lo solicitan.

El artículo 5 del Reglamento Interno de la CRUBC señala que la Comisión celebrará sesiones ordinarias y extraordinarias. Las sesiones ordinarias se realizarán trimestralmente y, las extraordinarias, cada vez que así lo determine el Intendente o mediante solicitud realizada por seis integrantes de la Comisión al Intendente. Sin embargo, esto último no es una práctica recurrente.

\section{Diversidad de participantes de la Comisión Regional de Uso de Borde Costero de la Región del Bío-Bío}

La CRUBC del Bío-Bío posee miembros de diversas afiliaciones. Tal como indica la Tabla 1, de un total de 49 integrantes estos están vinculados en mayor medida al Gobierno y sector público, luego a pueblos originarios, sigue el sector pesquero artesanal/industrial y en igual medida están representados 
el sector turístico, portuario y científico. No existen miembros relacionados o vinculados a grupos u organizaciones ambientales.

Tabla 3. Afiliación de actores de la Comisión Regional de Uso de Borde Costero de la Región del Bío-Bío

\begin{tabular}{|c|c|c|c|c|c|c|c|c|c|}
\hline & Gobierno & $\begin{array}{l}\text { Universidad/Con } \\
\text { sultor Científico }\end{array}$ & $\begin{array}{c}\text { Representantes } \\
\text { pesca artesanal } \\
\text { e inustrial }\end{array}$ & $\begin{array}{l}\text { Representantes } \\
\text { Sector Turismo }\end{array}$ & $\begin{array}{l}\text { Representantes } \\
\text { Sector Acuícola }\end{array}$ & $\begin{array}{l}\text { Grupos/Organizaci } \\
\text { ones Ambientales }\end{array}$ & $\begin{array}{c}\text { Pueblos } \\
\text { Originarios }\end{array}$ & $\begin{array}{c}\text { Sector } \\
\text { Portuario }\end{array}$ & $\begin{array}{l}\text { Puntuacion de } \\
\text { Diversidad de } \\
\text { Afiliación } \\
\text { (Basandose en } \\
\text { el Índice de } \\
\text { Blau) }\end{array}$ \\
\hline CRUBC Región del Bio Bío & 33 & 2 & 4 & 2 & 1 & 0 & 5 & 2 & 0.52 \\
\hline
\end{tabular}

Según el Índice de Blau, la CRUBC del Bío-Bío es moderadamente heterogénea ya que el resultado indica estar solo un poco por el sobre a la mitad entre el valor 0 (completamente homogéneo) y el valor 1 (heterogenia máxima). Se visibiliza que 33 de 49 miembros corresponden a actores públicos.

Cabe señalar que el artículo número 3 del Reglamento Interno de Funcionamiento de la Comisión Regional de Uso de Borde Costero de uso del Borde Costero de la República contempla a lo menos a nueve representantes de los sectores no provenientes del gobierno o de la administración pública. Estos son:

- Dos representantes del sector pesquero artesanal

- Dos representantes del sector acuícola

- Dos representantes del sector turístico

- Tres representantes de otros sectores, diferentes de los señalados precedentemente, nombrados por el Intendente Regional.

Se puede observar que, la Región del Bío-Bío tiene en un total de 16 miembros que no representan al Gobierno/Administración Pública, por lo que es considerablemente más de lo que contempla el propio reglamento ya que este solo contempla a nueve. En este sentido, la CRUBC de la Región del Bío-Bío ha propiciado la inclusión de actores a la Comisión, generando una mayor diversidad que la contemplada en el propio reglamento. De lo anterior se puede inferir que, el borde costero es activo en la Región del Biobío y que además los interesados/as tienden a canalizar sus demandas a través de una participación por vía institucional como lo es la CRUBC.

Con respecto a la diversidad de creencias, éstas se definen para efectos de esta investigación, en las posturas que tienen los integrantes con respecto a la naturaleza y a las distintas visiones que tienen con respecto al borde costero y sus significados. Para los actores entrevistados el borde costero es relevante para el desarrollo de regional y reconocen la existencia de una diversidad de usos en él.

Los actores señalan que las problemáticas actuales del borde costero son la contraposición de usos del borde costero, la falta de una preponderancia de una regulación estatal y otros señalan que, los plazos de los permisos para ocupar el borde costero son muy extensos y obstaculizan la realización de las actividades económicas en este espacio, tanto en el caso de solicitud de permisos a través de concesiones marítimas como de solicitud de Espacios Marino Costero para Pueblos Originarios.

En este sentido, se observaron opiniones diferentes y es posible inferir una diversidad de creencias sobre el territorio costero toda vez que un actor identifica el territorio como una oportunidad de desarrollo económico para la región y otros que aluden a una falta de regulación estatal más estricta y ordenada para la regulación de sus usos. 


\section{Aprendizaje social en la Comisión de Uso de Borde Costero}

Según la percepción de los miembros, la CRUBC es un espacio que sirve para conocer el quehacer de los otros actores en el borde costero con respecto de sus actividades y opiniones. Asimismo, es un espacio que sirve para mostrar a los otros lo que hace determinado gremio, buscando sensibilizar ciertas posturas.

Sin embargo, es posible inferir que este aprendizaje no alcanza para lograr consensos ya que, algunos entrevistados señalan que en las sesiones el comportamiento se asemeja a un "diálogo de sordos" por lo que es muy difícil que las personas neutralicen o cambien sus opiniones.

Con respecto a los conocimientos adquiridos según los aspectos técnicos, económicos y legales del borde costero, es posible visualizar una percepción positiva, teniendo una predominancia los aspectos legales del borde costero, por lo que es posible señalar que la CRUBC ha sido un espacio de aprendizaje sobre todo en los términos legales del borde costero.

Se deduce que ha existido un mayor aprendizaje cognitivo que relacional al interior de la CRUBC, ya que predominan visiones poco conciliadoras y a su vez alto aprendizaje normativo/legal. Nudos críticos del aprendizaje social en la CRUBC

\begin{tabular}{|c|c|c|}
\hline EQUIDAD PROCESAL & CONOCIMIENTO CIENTÍFICO & PARTICIPACIÓN \\
\hline 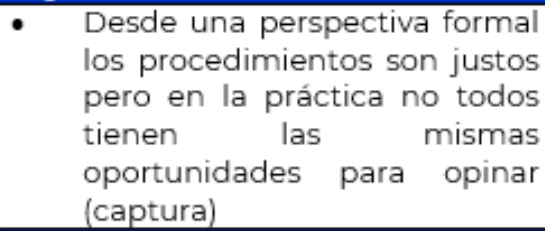 & $\begin{array}{l}\text { - Existe una percepción de } \\
\text { carencia de conocimiento } \\
\text { científico para la toma de } \\
\text { decisiones. }\end{array}$ & $\begin{array}{l}\text { - } \quad \text { Existe demanda externa } \\
\text { por participar en la } \\
\text { CRUBC en la Región del } \\
\text { Biobío no así en las otras } \\
\text { regiones consultadas. }\end{array}$ \\
\hline CONFIANZA INTERPERSONAL & LIDERAZGO RESPETADO & PERCEPCIÓN DE CONFLICTO \\
\hline $\begin{array}{l}\text { - } \\
\text { Existen desconfianzas por } \\
\text { algunosta miembros (alcaldes) } \\
\text { En discusión sobre concesión } \\
\text { marítima de terminal de gas } \\
\text { GNL Talcahuano es posible } \\
\text { notar una dualidad de opinión } \\
\text { y de votos disidentes entre los } \\
\text { alcaldes y los otros actores. }\end{array}$ & $\begin{array}{l}\text { - } \quad \text { El liderazgo no es ejercido por } \\
\text { la figura del Intendente. } \\
\text { Entrevistados señalan las } \\
\text { siguientes características de } \\
\text { liderazgo necesarias: } \\
\text { transparencia, apertura, } \\
\text { empatía y humildad. } \\
\text { El } 50 \% \text { de los entrevistados } \\
\text { confirma que existe a lo menos } \\
\text { un participante que media el } \\
\text { conflicto y que es respetado } \\
\text { por todos. }\end{array}$ & $\begin{array}{l}\text { - Los miembros no } \\
\text { consideran a la CRUBC } \\
\text { como una instancia } \\
\text { particularmente } \\
\text { conflictiva. } \\
\text { Los miembros de la } \\
\text { CRUBC consideran más } \\
\text { conflicto el borde costero } \\
\text { que la CRUBC en } \\
\text { particular. }\end{array}$ \\
\hline
\end{tabular}

\section{Obstáculos y fortalezas de la CRUBC para la gestión del borde costero en Chile}

Al momento en que los miembros fueron consultados sobre los nudos críticos que debían enfrentar para la gestión del borde costero en la CRUBC, señalaron principalmente la importancia de contar con el instrumento de zonificación territorial aprobado. En efecto, lo anterior se puede considerar uno de los mayores obstáculos que tienen las regiones que no cuentan con Zonificación de Uso de Borde Costero, cuyas causas se relacionan con la falta de orientación y recursos para la culminación de este proceso. Además, los entrevistados hicieron referencia al procedimiento de toma de decisiones, considerando que éstas debiesen ser vinculantes. 


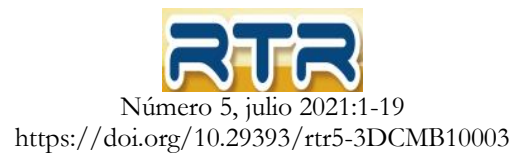

Nosotros creemos que las decisiones perfectamente se pueden tomar en la región no solo emitir una opinión, sino que la comisión resuelva si es el mejor uso, por los fundamentos que sea, otorgar esa concesión, ese permiso, entonces el particular desarrollaría su proyecto en un tiempo muchos más breve (Entrevista con coordinador regional Región del Bío-Bío, comunicación personal, 2020).

Con respecto al funcionamiento interno, cabe señalar que existen críticas por parte de los miembros, ya que la CRUBC no es considerada un espacio para lograr consensos, destacándose por la urgencia y la premura de los votos antes de hacer una gestión planificada y estratégica del borde costero. Lo anterior es posible relacionarlo a que las funciones de la CRUBC se han reducido solamente al trámite de concesiones marítimas por sobre la implementación de la Política Nacional de Uso de Borde Costero.

Es más importante el análisis y no la premura y no la urgencia. Mejor demorarse una semana, dos semanas más que estar con el tiempo ahí y presionando que la votación tiene que ser ahora porque se acaba el tiempo (Entrevista con representante sector académico Región del Bío-Bío, comunicación personal, 2020).

Además, los actores perciben que la diversidad si es un problema a la hora de hacer gestión del borde costero. Sin embargo, los miembros también destacan que es una fortaleza el hecho de contar con un espacio donde los actores tanto públicos como privados puedan informarse y manifestar su opinión.

Otro obstáculo identificado tiene relación con la institucionalidad ambiental, donde los actores no tienen claridad sobre cuál es su rol frente a las problemáticas ambientales que conlleva aprobar o rechazar determinados proyectos.

En este sentido, existen actores que señalan que el rol de la CRUBC no debiese tener relación con pronunciamientos u observaciones medioambientales ya que existen otras instancias que velan por el cumplimiento de la normativa ambiental vigente. Otros actores señalan que la incidencia de la CRUBC se ve afectada debido a que los proyectos finalmente se aprueban en otras instancias, por lo que la CRUBC solo vendría a ser un espejo de lo que el sistema medioambiental ya ha decidido.

Nosotros como CRUBC tenemos que hacernos parte de la discusión en el sistema medioambiental, o llegamos después. Cuando llegamos después no tiene mucho sentido. ¿Entonces en qué momento la CRUBC toma más fuerza? (Entrevista con Coordinador Regional, comunicación personal, 2020).

Además, los proyectos ya están aprobados en otras instancias como el SEIA. Por eso es un saludo a la bandera porque la concesión, el proyecto ya está aprobado en otras instancias... (Entrevista con coordinador regional, comunicación personal 2020).

Las fortalezas identificadas corresponden a la valorización positiva que realizan los miembros de la CRUBC con respecto de contar con una instancia multiactor donde actores estatales y no estatales tengan derecho a participar, a pesar de que esto les signifique complejidades para la generación de consensos. 


\section{Conclusiones}

La gobernanza colaborativa promete resultados positivos en la implementación de políticas públicas y una ampliación de la participación democrática. Sin embargo, según el caso estudiado ha sido posible concluir que tal como plantea la literatura revisada, existen elementos que impiden el aprendizaje social, afectando las decisiones y los resultados logrados por la CRUBC. Los resultados sugieren que el funcionamiento de la gobernanza de la CRUBC depende del marco institucional y el liderazgo ejercido.

En relación con la diversidad institucional de los miembros, en el caso de la Región del BíoBío, la participación es amplia, ya que considera una mayor cantidad de actores no estatales en su nómina de miembros que la que exige el propio reglamento. A mayor diversidad se puede complicar el logro de acuerdos y se requiere un mayor esfuerzo de gestión y liderazgo para poder llegar a resultados de gobernanza positivos.

Los miembros entrevistados valoran las iniciativas con altos niveles de participación y diversidad, para poder conocer el trabajo y actividades que se están desarrollando en el borde costero, pero no se obtienen consensos sólidos. Además, los miembros de la CRUBC han manifestado que la instancia es una audiencia donde cada quien puede manifestar su opinión, pero, la premura del tiempo y el acceso a la información muchas veces hacen que el foro se transforme en un "diálogo de sordos". Se puede deducir que, faltan procedimientos intermedios para el trabajo en equipo que propenda a la construcción de confianzas y la legitimación de posturas y la construcción de acuerdos.

Con todo, la CRUBC es un buen espacio para que los interesados se conozcan y además interactúen sobre los diferentes puntos de vista con respecto de los distintos usos del borde costero. $\mathrm{Al}$ mismo tiempo, no se observa el establecimiento eficaz de una meta en común entre los actores.

En relación con los conflictos, un aspecto relevante en todas las Comisiones consultadas, es que las discusiones tiendan a cumplir un rol fiscalizador ambiental de los proyectos consultados a la CRUBC. No obstante, no se observa que han logrado construir confianzas a través de procedimientos formales que equilibren los aspectos técnicos con los políticos. En este sentido, existen visiones opuestas del rol que tiene la CRUBC sobre cuál aspecto de la compatibilidad de usos del borde costero es el que se debe abordar y discutir en la Comisión.

El punto anterior es problemático ya que hace alusión al conocimiento que tienen los miembros sobre los "protocolos básicos y las reglas básicas de colaboración y que son fundamentales para la legitimidad del proceso" (Ansell \& Gash, 2007, p.24).

Un aspecto positivo a destacar es la incorporación de actores de acuerdo a la actualización de los intereses en el borde costero, así como se ha hecho con el sector energético, turístico y los pueblos originarios, ya que "el éxito de la colaboración depende de la inclusión de un espectro lo suficientemente amplio de interesados para reflejar el problema" (Gray, citado en Ansell y Gash, 2007, p.25). Esta última afirmación incluye a los actores más problemáticos o conflictivos.

Otro aspecto relevante del diseño institucional, es la exclusividad que posee la CRUBC con respecto a la gestión del borde costero. Según la percepción de los participantes no es posible confirmar esta exclusividad debido a que existe una baja intersectorialidad con otras instancias ambientales, pero también con los sectores de inversión pública², por lo que esto podría bajar los incentivos de los participantes, ya que el foro podría verse como poco incidente o relevante en materias de borde

\footnotetext{
${ }^{2}$ La CRUBC no tiene injerencia en otros instrumentos de planificación propios del borde costero como lo es el Plan Intercomunal de Borde Costero del Ministerio de Vivienda y Urbanismo. Con respecto a esto, una de los miembros de la CRUBC señala que con respecto a instrumentos de ordenamiento territorial que se vinculan con el borde costero, él ha participado más en otras instancias que en la propia Comisión.
} 
costero. Además, podría movilizar a los actores excluidos a participar más activamente en otras instancias o crear otras distintas a la institucionalidad vigente.

Aunque el análisis realizado no permitió determinar si la diversidad de la CRUBC ha sido beneficiosa para el aprendizaje social entre los miembros, sí permite algunas observaciones. Primero, la diversidad de actores es valorada por los miembros particularmente como una buena fórmula para incluir a los interesados y conocer las actividades que realizan otros actores. Segundo, el proceso colaborativo de la CRUBC no es capaz de moderar la relación entre la diversidad y el aprendizaje social ya que no existen procedimientos de funcionamiento que contribuyan al trabajo en equipo y la conformación de consensos.

En el caso de la conflictividad de la CRUBC no es posible determinar con suficiente fuerza una relación antagónica que obstaculice el aprendizaje. Sin embargo, existen creencias de algunos participantes de una utilización política de la CRUBC por parte de alguno de sus miembros.

\section{Propuestas de Mejoras}

Para mejorar los resultados de gobernanza colaborativa y aumentar el aprendizaje social entre los miembros se insta a una mayor autonomía de la CRUBC en la toma de decisiones ya que esto incrementaría los incentivos de participación y el compromiso de los participantes. Además, sería necesario aumentar la cantidad de sesiones en base a una agenda construida por todos los miembros ya que esto podría aumentar la confianza entre ellos.

En relación a la confianza y aprendizaje social requerido para la gobernanza, se recomienda instaurar procedimientos intermedios entre las discusiones y el voto, como por ejemplo la coconstrucción de agendas, ya que la elaboración según la demanda de solicitudes de concesiones marítimas y de Espacios Marítimos Costeros para Pueblos Originarios puede ocasionar un detrimento en la confianza de los participantes. La co-construcción de agenda entre todos los actores puede contribuir a evitar una utilización del espacio para buscar votos y propender a recoger iniciativas, deseos e intereses de los otros participantes, superando el funcionamiento en base a demanda y a la solicitud desde el nivel central. Esto último podría desarrollar una mayor capacidad propositiva de la CRUBC.

Además, es necesario cambiar el enfoque de la colaboración, centrándose en la Política Pública y la gestión del borde costero más que en los hechos puntuales de negociación que generalmente propenden a tener una visión desintegrada y a un detrimento de visiones y metas conjuntas.

En cuanto al ámbito de la capacidad institucional de la Comisión, falta mayor atribuciones para poder gestionar de mejor manera la diversidad de la CRUBC tanto en la Región del Bío-Bío como en las otras regiones.

A pesar de que en la Región del Bío-Bío no se mencionaron problemas de dotación de personal ni recursos, es posible visualizar que al igual que las otras regiones, tiene inconvenientes en implementar la Política Nacional de Uso de Borde Costero, así como también en la consolidación del instrumento de zonificación de uso de borde costero. Una solución podría ser una mayor dotación de recursos y una mayor autonomía para mejorar los incentivos de participación de los miembros y un liderazgo que proponga objetivos y metas comunes entre los participantes.

\section{Agradecimientos}

Agradezco el apoyo financiero de INCAR a través del proyecto ANID-FONDAP No. 15110027. 


\section{Bibliografía}

Aguilar, LF. (2016). Las transformaciones posburocráticas, posgubernamentales y transterritoriales del Estado y del gobierno. Sociologica, 31(89), 9-43. Obtenido de: http://www.scielo.org.mx/pdf/soc/v31n89/0187-0173-soc-31-89-00009.pdf. [2020, 14 de junio]

Altschuler, B. (2013). Territorio y desarrollo: aportes de la geografía y otras disciplinas para repensarlos. Revista Theomai (27-28), 64-79. Obtenido de: http://revistatheomai.unq.edu.ar/numero_27-28/altschuler.pdf. [2020, 03 de septiembre]

Alfie, M. (2013). Democracia deliberativa y gobernanza ambiental: ¿conceptos transversales de una nueva democracia ecológica? Revista Scielo, 28(80), 73-122. Obtenido de: http://www.scielo.org.mx/scielo.php?script=sci_arttext\&pid=S0187-

$01732013000300003 \& \operatorname{lng}=$ es\&tlng=pt. [2020, 15 de julio]

Ansell, C. \& Gash, A. (2008). Collaborative Governance in Theory. Journal of Public Administration Research and Theory 18(4), 543-571. Obtenido por:

https://doi.org/10.1093/jopart/mum032 [2020, 12 de abril]

Barragán, J.M. (coord.). 2011. Manejo Costero Integrado y Política Pública en Iberoamérica: Propuestas para la acción. Cádiz, España: Red IBERMAR (CYTED),

Barragán, J.M. (2014). Política, gestión y litoral: Una nueva visión de la gestión integrada de áreas litorales. Madrid, España: Tébar Flores.

Bolados, P. (2016). Conflictos socio-ambientales/territoriales y el surgimiento de identidades post neoliberales (Valparaíso-Chile). Izquierdas, (31), 102-129. https://dx.doi.org/10.4067/S071850492016000600102 [2020, 23 de noviembre]

Brugué, Q., Canal, P., Paya, P. (2015). ¿Inteligencia administrativa para abordar "problemas malditos"? El caso de las comisiones interdepartamentales. Gestión y política pública, 24(1), 85-130. Obtenido de:

http://www.scielo.org.mx/scielo.php?script=sci_arttext\&pid=S140510792015000100003\&lng $=$ es\&tlng $=$ es $[2020,19$ de octubre $]$

Canto, R. (2012). Gobernanza y democracia: De vuelta al río turbio de la política. Gestión y Política Pública, 21(2), 333-374. Obtenido de:

http://www.scielo.org.mx/scielo.php?script=sci_arttext\&pid=S1405-10792012000200002.

[2020, 03 de marzo]

CEPAL, (1996). La política de borde costero como un instrumento de desarrollo sostenible: análisis desde la normativa ambiental internacional y regional. Obtenido de: https://repositorio.cepal.org/handle/11362/19587 [2020, 30 de agosto]

Delamaza, M. (2017). Consecuencias políticas de los conflictos socio-territoriales en América Latina. Conceptos y perspectiva comparada. En Obtenido de:

http://www.congresoalacip2017.org/arquivo/downloadpublic2?q=YToyOntzOjY6InBhcmFt cyI7czozNToiYToxOntzOjEwOiJJRF9BUIFVSVZPIjtzOjQ6IjI3MjkiO30iO3M6MToiaCI7czozMjoi N2I0Mzg3MzNkNTE3ZTA4NTc3YTYzYTZkOGJiZGI1NzUiO30\%3D [2020, 04 de octubre]

Flores, P. (2016). La Comisión Regional del Uso del Borde Costero: Régimen jurídico en el procedimiento administrativo de otorgamiento de concesiones maritimas (Tesis de posgrado). Santiago: Universidad de Chile. Obtenido de: http://repositorio.uchile.cl/handle/2250/144198 [2019, 20 de diciembre]

Gordon, S. (2005). Confianza, capital social y desempeño de organizaciones. Criterios para su evaluación. Revista mexicana de ciencias políticas y sociales, 47(193), 41-55. Obtenido de http://www.scielo.org.mx/scielo.php?script=sci_arttext\&pid=S0185-

$19182005000100041 \& \operatorname{lng}=$ es\&tlng=es. [2020, 06 de junio]

Gudynas, E. (2015). Extractivismos. Ecología, economía y política e un modo de entender el desarrollo y la naturaleza. Obtenido de: 
http://gudynas.com/wpcontent/uploads/GudynasExtractivismosEcologiaPoliticaBo15Anunci o.pdf. 2020, 17 de noviembre]

Hernández, F., Rivera, J. (2017). Gobernanza, ciudades y políticas públicas, una conversación a propósito de Hábitat III y la Nueva Agenda Urbana. Entrevista con Joan Subirats. Revista Latinoamericana de Políticas y Acción Pública. 2(3), 101-113. Obtenido de: https://revistas.flacsoandes.edu.ec/mundosplurales/article/view/2846. [2019, 20 de diciembre]

Leary, O., Vij, N. (2012). Collaborative Public Management Where Have We Been and Where Are We Going? The American Review of Public Administration. [2020, 03 de julio]

Martínez, C., Arenas, F., Bergamini, K., Urrea, J. (2019). Hacia una ley de costas en Chile: criterios y desafíos en un contexto de cambio climático. 42(5), 507-522. Obtenido de: https://www.researchgate.net/publication/258126421_Collaborative_Public_Management_Where_Ha ve_We_Been_and_Where_Are_We_Going/citations [2020, 05 de febrero]

Ministerio de Defensa (1994, 14 de diciembre). Decreto 475 Establece la Política Nacional de Uso de Borde Costero del Litoral de la República, y crea la Comisión Nacional que Indica. En Biblioteca del Congreso Nacional de Chile [en línea]. Recuperado de

https://www.ssffaa.cl/pdf_documentacion/cnubc/normativas_y_reglamentos/5_cnubc/politi ca_nacional_uso_borde_costero_DS475.pdf. [2019, 01 de diciembre]

Ministerio de Defensa (2005, 28 de febrero). Oficio Gabinete Presidencial No001 Reglamento Interno de Funcionamiento de la Comisión Regional de Uso del Borde Costero de la República. En Subsecretaria para las Fuerzas Armadas [en línea]. Recuperado de http://www.ssffaa.cl/pdf_documentacion/cnubc/normativas_y_reglamentos/5_cnubc/OF_GAB_00 1_2005_establece_nuevo_Reglamento_CRUBC.pdf. [2019, 01 de diciembre]

Morales, C. (2009). La Diversidad en las Organzaciones ¿De qué estamos hablando? Manegement and Society, 9, 16-19. Obtenido de

https://www.researchgate.net/publication/319482661_La_diversidad_en_las_organizaciones_ De_que_estamos_hablando [2020, 22 de marzo]

Observatorio Laboral del Biobío (2018). Realidad Laboral y Económica de La Región del Biobío. Obtenido de:

http://observatoriobiobio.cl/AdministracionOL/ESTUDIOS/Estudio_Realidad\%20laboral\% 20y\%20economica_OLB_2018.pdf. [2020, 15 de octubre]

Parrado, S. (2017). La gobernanza colaborativa y la teoría de los bienes comunes. En C. Colino,

Ciencia y política, una aventura. Valencia: Tirant Lo Blanch. Obtenido de: https://www.academia.edu/36016088/PARRADO-2017-

Gobernanza_colaborativa_y_teor\%C3\%ADa_de_los_bienes_comunes.pdf [2019, 04 de noviembre]

Ramírez, M. (2011). Gobernanza y legitimidad democrática. Reflexión Política, 13(25), 124-135. Obtenido es: http://www.redalyc.org/pdf/110/11018897010.pdf [2019, 16 de diciembre]

Quezada, M (2019). Gobiernos locales con gobernanza interactiva: El caso del programa escuela abierta de la Municipalidad de Recoleta. Revista Territorios y Regionalismos, (1).

Siddiki, S., Kim, J., \& Leach, W. (2017). Diversity, Trust and Social Learning in Collaborative Governance. Public Administration Review, 77, 863-874.

Subsecretaría para Las Fuerzas Armadas. (20 de diciembre de 2019). Subsecretaría para Las Fuerzas Armadas. Obtenido de https://www.ssffaa.cl/asuntos-maritimos/zonificacion-del-bordecostero/zonificacion-region-de-araucania/

Torre, A. (2016). El rol de la gobernanza territorial y de los conflictos de uso en los procesos de desarrollo de los territorios. Revista Geográfica de Valparaiso, (53), 07-22.

Universitat Politècnica de València. (2015, 30 de julio). Buenas Prácticas Científicas [video]. YouTube https://www.youtube.com/watch?v=EDKz76SVdzU [2019, 03 de noviembre] 
Vásquez, A. (2010). El enfoque de la Gobernanza en el estudio de la transformación de las Políticas Públicas: Limitaciones, retos y oportunidades. Estudios de Derecho, 67(149), 246-260.

Wanna, J. (2008). Collaborative government: Meanings, dimensions, drivers and outcomes. In J. O'Flynn \& J. Wanna (Eds.), Collaborative governance a new era of public policy in Australia? Canberra: The Australian National University Press. Obtenido de:

https://www.researchgate.net/publication/331186677_Collaborative_government_meanings_ dimensions_drivers_and_outcomes/comments. [2020, 05 de julio] 